\title{
Research on Modification and Optimization of Network Topology and Internet Security based on Neural Network Structure and Mathematical Optimization
}

\author{
Gaoli Zhao ${ }^{1,2}$, Junping Song ${ }^{1}$ and Wei Chen ${ }^{2}$ \\ ${ }^{1}$ Henan Institute of Science and Technology, Henan, Xinxiang, 453003 \\ ${ }^{2}$ Wuhan University of Technology, Hubei, Wuhan, 430070 \\ zhaogaoli2015@126.com
}

\begin{abstract}
With the rapid and bursting development of computer science and related technology, the design and optimization of network topology has been a most important issue for thee researchers to solve. However, the increasing needs of the current control schemes have already been shown. In this paper, we theoretically analysis the features of neural network structure and convex optimization related method. There are a lot of shortcomings but the cable network should be restricted the wiring in some situations, for example, wiring, reroute workload big, easy to damage, in the network, each node cannot move and so on. With the mathematical optimization, game theory, mathematical economics and optimal control theory to the development of disciplines, convex analysis are increasingly brought to the attention of the people. Our proposed methodology combines the neural network and optimization theory to obtain better performance, the experimental result shows the performance of our approach compared with other state-ofthe-art algorithms.
\end{abstract}

Keywords: Network Topology; Neural Network; Mathematical Optimization; Convex Set and Theory; Modification and Optimization; Network Security

\section{Introduction}

With the bursting development of computer science and the broad applications of Internet, the design and optimization of network topology has been a most important issue for the researchers to solve. However, the increasing needs of the current control schemes have already been shown. Usually computer network transmission medium is mainly copper or fiber optic cable to constitute a wired LAN. There are a lot of shortcomings but the cable network should be restricted the wiring in some situations, for example, wiring, reroute workload big, easy to damage, in the network, each node cannot move and so on [1]. Currently, centering on the server set line network system provides the network interconnection of realistic possibility, but also become the bottleneck of influence and restrict network; Huge amounts of information rich web content but the development of multimedia technology such as images, sounds, makes the network operation of puff; No gold tower headless network authority easy to expand but along with its growing increasingly exposed its bloated and fragile. Therefore, to raise the comprehensive optimization of network performance has become the inevitable and necessary result of the development of network development [2]. Neural network is imitating people cranial nerve system information processing model of intelligent system which is some simplification process, abstraction and simulation of neural network. Its main features are: the massively parallel processing and distributed information storage, good adaptive, selforganizing and strong learning function, flexible function and fault tolerance. The neural network in image processing, pattern recognition, speech synthesis and intelligent robot has achieved gratifying results which becomes the world's most challenging and the most 
intriguing research hot spot [3]. Computer research computer network reliability model town by complexity as a system engineering science, after more than 50 years of development, it has formed a relatively complete and sound system. The principle of the computer network reliability is defined as: under the conditions stipulated in the computer network, within the stipulated time, network connected and satisfies the requirement of communication ability. Call it the computer network reliability. It reflects the computer network topology to support normal operation ability of the computer network [4]. The computer network reliability problem is to the reliability of the model into a figure. Computer network model using probability diagram to express the issue which is denoted as: $G(v, E)$. Computer network model of probability graph is the figure of the edge and the normal operation of the node state endowed with a certain probability value of figure in the future. Figure the reliability problems of contains two aspects: one is to analyze problems, namely calculate the reliability of a given threshold; the second, it is the design problem, namely all elements in a given design has the biggest reliability figure [5]. The design principle of the computer network reliability in computer network design and construction of engineering practice, scientific research personnel summarize a lot of specific design experience and the principle, the computer network reliability optimization design has played a good specification and guidance.

There has been large number of research institutions focusing on the research of optimizing the network topology. In [6], Kory's research group conduct review on the topic of topology optimization. In their research, they provides a literature review on transmission control and discusses current industry practices involving transmission control; the goal of their research is to reemphasize the importance of transmission control in order to initiate future research. The main concern is no longer in the design of the computer network topology optimization, the distribution of the limited channel capacity, and the flow distribution to make the cut in all channels have uniform load in order to minimize the average time delay problem. Because the construction and maintenance of the communication channel is more expensive which should make full use of the letter and don't make it waste, and the computer network link capacity requirements has become the key problem of optimization design. In [7], Salman et al. introduced a novel fuzzy particle swarm optimization (FPSO) algorithm for network topology optimization approach. As discussed popular in the research community, particle swarm optimization (PSO) is a powerful optimization technique that has been applied to solve a number of complex optimization problems. For the optimization design of the computer network is a multi-objective optimization problem which is harder to find the best solution. In order to simplify the design, the prominent main factors, ignoring secondary factors, the method of the computer network design problem is simplified to a variety of programming problem. Some of the early algorithm considering the topology optimization and the flow distribution is more and more as the heuristic algorithm which can't get the optimal solution already cannot satisfy the demand of the current computer network design. Their proposed fuzzy PSO algorithm is empirically evaluated through a preliminary sensitivity analysis of the PSO parameters which gains better optimal solution for the issues. Moreover, in [8], Elias's research group undertakes the research on scalable diffusionaware optimization. In the progress of computer technology and the rapid development of communication network, has made the optimal design of the computer network link capacity requirements become the key issues in computer network design. To this end, this paper proposes a computer network link capacity optimization design method and the overall optimal solution can be obtained. Therefore, in the network design, the total cost of the link is usually viewed as a network of the total cost. Number of edges on behalf of the network topology structure, that is, the configuration of nodes and links, configuration node equipment should be in what position each node should ask what is the links. The remaining question is asked which nodes should have a link. More related literature reviews could be found in the articles [9-15]. 
Therefore, to obtain solution for the challenges mentioned and look for more optimal solution for the optimization problems. We theoretically analysis the features of neural network structure and convex optimization methodology to obtain better result.

\section{Related Knowledge and Prior Research}

\subsection{The Principles of Deep Neural Network}

As the core reference of our methodology, deep and modified neural network theory serves as the foundation of our approach. Artificial neural network system is a kind of engineering computation model which is composed of a large number of neurons is connected. In general, each neuron corresponds to an incentive function and every two connected the weights between neurons. Based on error back algorithm of BP neural network is a kind of research is relatively mature and wide application of artificial neural network. Because this kind of neural network structure is simple, and can effectively nonlinear objective function approximation, and therefore is widely used in pattern recognition and other disciplines and fields. Traditional BP algorithm is composed of signal is spread and error back propagation of two processes, namely: (1) the sample input from the input layer, the hidden layer after processing, the output from the output layer; (2) based on neural network error between actual output and the expected output of the negative gradient direction, from a backward step by step to iterative correction between neurons in each layer connection weights and threshold of neurons [16]. The following figure 1 shows the organization of the neural network.

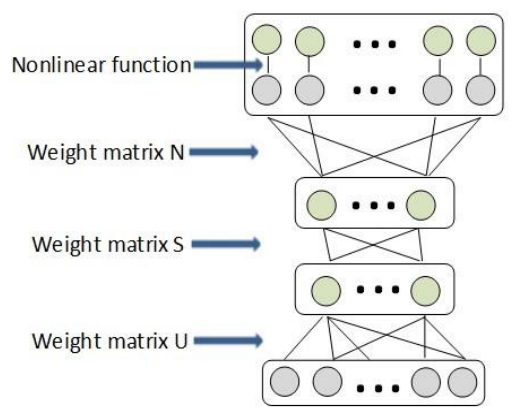

Figure 1.The General Organization of the Neural Network

Traditional BP algorithm has some inherent defects, such as easy to fall into local minimum point, slow learning speed and more importantly, the optimal network structure is difficult to determine [17]. The following formula shows the cost function.

$$
\Phi=\lambda_{r} \sum_{t} \sum_{i} E_{r}\left(J_{i}^{(t)}, J_{i}^{(t)}\right)+\lambda_{d} \sum_{t} \sum_{p} \sum_{l} E_{d}\left(J_{i}^{(t)}, J_{i}^{(t)}\right)
$$

Most current classification, regression, such as learning method for shallow structure algorithm and the limitation lies in limited samples and cell cases of complex function said ability is limited, its generalization ability for complex classification problems under certain constraints. The formula 2 shows the feature.

$$
v(i)=f_{a c t}\left(\sum_{j \in R_{i}} w_{i, j} v(j)\right)
$$

Inspired by the brain structure layers, neural network researchers have been trying to multilayer neural network research. Gradient descent of BP algorithm is a 
classical and uses the random initial value of multilayer network training algorithm is selected, but because of the nonlinear mapping between the input and output to make network error function or energy function space is a nonlinear space containing multiple minimum point. The revised structure of the neural network is shown in the Figure 2.

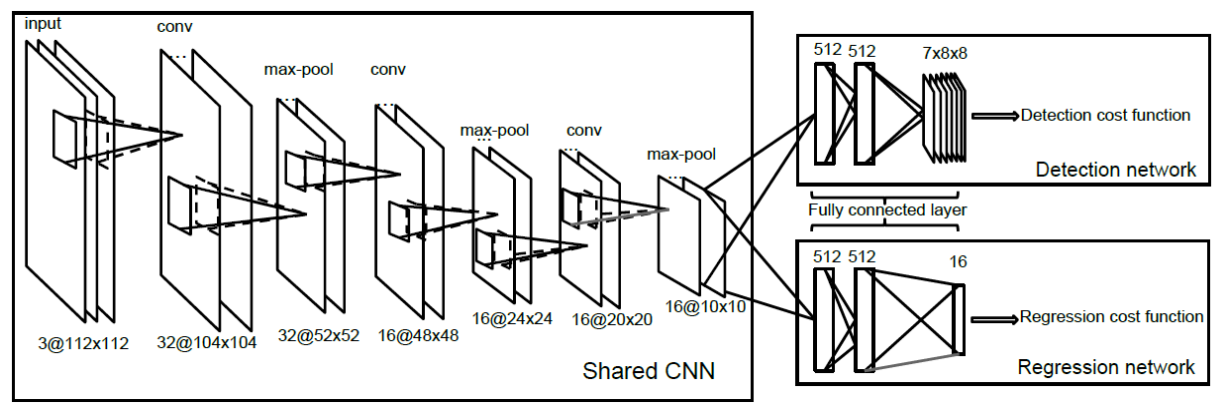

Figure 2.The Revised Structure of Modified Neural Network

Based on template matching model with two layers of the cell can be thought of, the first layer building to multiple template matching of input data, each unit can output a match; The second in particular mechanism, the first layer of output matching degree. A typical example is based on local matching method. Kernel methodology is always adopted which could be found in the literatures [18-25]. The following formulas introduce the feature.

$$
\begin{aligned}
& f(x)=b+\sum_{i} \alpha_{i} K\left(x, x_{i}\right) \\
& \min _{\alpha} \frac{1}{2}\|\alpha\|_{2}^{2}+C \frac{1}{N} \sum_{i=1}^{N} \Delta\left(f\left(X_{i}\right), y_{i}\right)
\end{aligned}
$$

The deep structure of deep learning has multiple nonlinear mapping which can complete complex function approximation is one of the advantages of deep learning. Also available distributed said deep learning theory that can go through the step by step a learning algorithm for the main drive variables of the input data. The advantage is that through the deep learning of unsupervised training algorithm is completed and by generative training can avoid network function expression ability is too strong and fitting situation. In conclusion, adding the advantages of the neural network to the optimization process is necessary.

\subsection{The Concepts of Mathematical Convex Optimization}

Convex analysis, or convex set and convex function theory, is a relatively young branch of mathematics. In the 1930s did not appear systematically study of convex set. $40 \mathrm{~s}$ and the 50s of the 20th century, especially in the field of optimization after found many applications of convex set which will further promote the development of the theory. With the mathematical optimization, game theory, mathematical economics and optimal control theory to the development of disciplines, convex analysis are increasingly brought to the attention of the people. Need to emphasize that the optimization of predictive control and the traditional sense of the discrete optimal control, there's a big difference in the optimization of predictive control is usually a limited period of time the rolling optimization. At each sampling instant, optimize performance indicators will involve only from the moment have a limited time in the future and to the next sampling instant, the optimal time goes forward to a time point. Predictive control, therefore, is not the same with a global optimization performance index, but at every moment engraved with a relatively optimal performance index of the moment. Different time optimization 
performance index of relative form is the same but its absolute form is different. We could write the cost function in the formula 5.

$$
C_{k}(P)=\sum_{i=1}^{m_{k}}\left|r_{k}(i) c_{k}(P)-c_{k}^{\prime} P\left[w_{k}(i)\right]\right|^{2}
$$

The function of the prediction model is based on the history of the object information and future input to predict its future output, so the equation of state and the transfer function of these traditional models can be used as prediction model. For linear stability and step response, impulse response the nonparametric model, also can be directly used as predictive model. In addition, the model of nonlinear system denoted as the corresponding distributed parameter system, as long as have the function which can also be used in the forecast of this kind of system control when used as predictive model. We can increase on the system state and control input constraints, such constraint conditions are usually in the form of collection constraints which is shown in the following Formula 6.

$$
\text { constrain }\left\{\begin{array}{cc}
u_{k} \in U, & k=i, i+1, \ldots, i+N-1 \\
x_{k} \in X, & k=i, i+1, \ldots, i+N \\
x_{i+N} \in X_{f} \subset X &
\end{array}\right.
$$

At this point is given in the collection with or without constraint conditions necessary and sufficient conditions for the optimal solution of the optimization problem and the solution of the optimization problem can be optimized by ellipsoid algorithm to solve. The related detailed applications for our method will be shown in the next section.

\section{The Proposed Methodology}

Computer network interconnection, and realize the unimpeded transport of information is based on the huge network system. Network system has a very important role in the development and the significance, achieved the integration of the global network, efficient information interaction which is the future direction and tendency of the development of the computer to the Internet. In the computer network system, therefore, not only must carry on the network system architecture analysis, more need to build the whole network system. In the network architecture of oneself, the need for proper network optimization is the fact. Under the network environment and the local conditions, to adjust measures to local conditions of computer network optimization, it is very necessary. In fact, the network system is the process of communication. The mutual interactions and the computer network system is the complex multimodal information communication system. According to the theoretical analysis of communication system, information is the information transmission start, source coding, the information is then transmitted through transmission medium, the transmission to the receiver, decoding is required first, and then the receiver to receive the corresponding information. And the principle of computer network system is the same, is the single channel of communication system interactions, and then realize the huge information transmission system, gradually formed the network system. Advantages of network system is relatively obvious, the transmission and interactive performance is stronger which can realize the effective transmission of information and the ability to decode. In this proper and efficient way, the communication between each other is relatively convenient and quick. The general organization of network applications is shown in the Figure 3. 


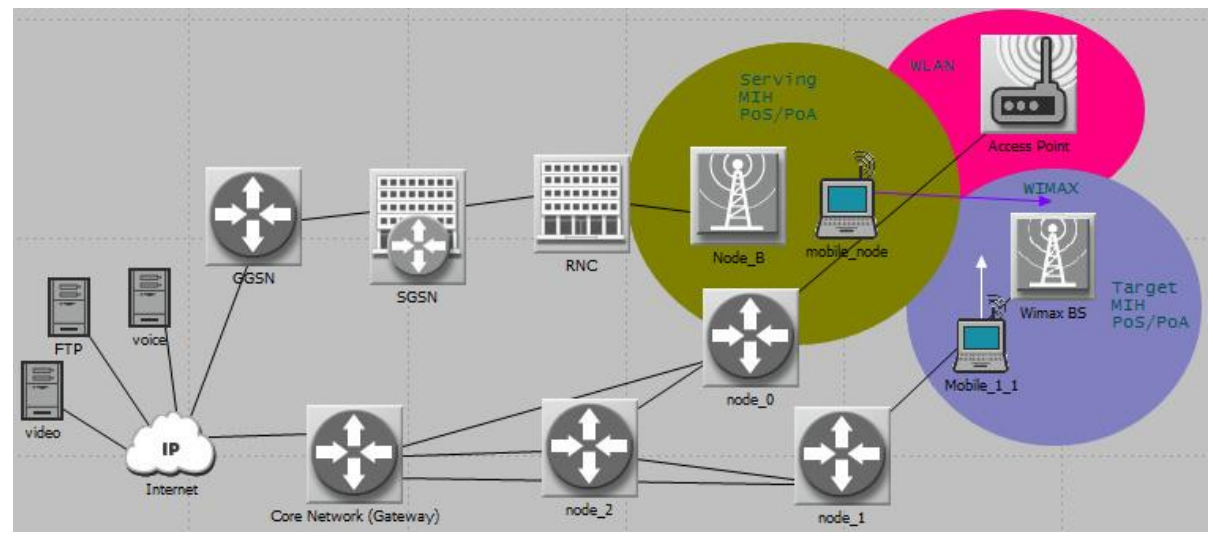

Figure 2.The General Organization of Network Applications

For the computer network system, set up successful, also need to adapt to the network optimization, to achieve maximization of resource utilization, so as to realize the reasonable sharing of network resources. In general, network optimization can be done from several aspects and the following is for common optimization methods are analyzed. (1) Network layer network optimization methods of structure analysis. Under normal circumstances, the network resources are relatively complex which need to classify different resource flow form processing to maximize the efficiency of network transmission. Therefore, for the computer network layered processing generally. In the physical layer and data link layer, generally deal with physical information, different host transmission of data, for example it will carry the physical address of the host. This is in order to be able to identify the source of information, and thus for the information security interaction. The Figure 3 shows the condition.

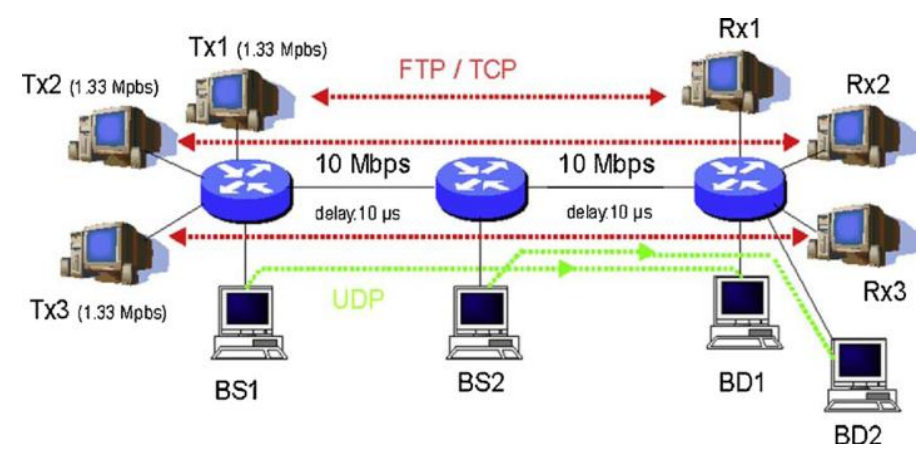

Figure 3.The Condition of Our Mentioned Network Organization

And really need to decode the usefulness of information, can enter the network layer, directly to receive information and interaction. In this way, we could realize the information interaction efficiency. At the same time, also is the network optimization is the most commonly used method. (2) Use the hardware such as switches way of optimization. Tree distribution mode of resource allocation, is one of the best way of resource allocation, for the computer network system of the allocation of resources, has a very important role. However, in some area, can use other ways of configuration, so that we can realize the better utilization of resources to a certain extent, so as to realize the optimization of the network, for example, within the local area network (LAN) we can adopt the way of radiation configuration, namely to a point as the center, and other equally host for radiant point. This configuration mode, can realize resource supply. Among them, we can make full use of the principle of resource allocation step by step [26-27]. In fact, it is will be biggest change in the distribution of network resources. (3) 
Cooperation between software and hardware configuration. The purpose of optimization is the ability to save network resources, but also can achieve the optimum use of the network. Two ways have their own advantages, to effectively combine, so it can be optimized that maximizes. The Figure 4 shows this condition.

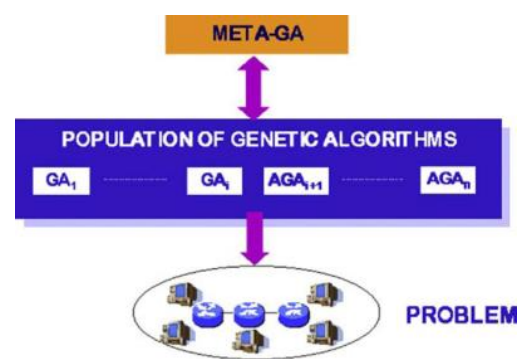

Figure 4.The Condition of the Proposed Scene

Network performance including transmission rate and throughput which is based on a number of factors for the performance of the wireless LAN, wireless local area network (LAN) equipment because of using different criteria, the hardware structure and software function is different, have different network performance. In addition to the above said problem can affect the performance of the wireless local area network (LAN), in the process of optimization of network performance, also may encounter four problems are: coordinate positioning, near-far effect, hidden sites and roaming between networks. Parallel genetic algorithm model can be divided into three categories: master-slave model, model of fine-grained and coarse-grained model L among them, the coarse-grained model is also called a distributed model or island, is the most widely and most adaptable coarsegrained parallel genetic algorithm model $\mathrm{L}$ model is in accordance with the number of processors will be randomly generated initial groups divided into several sub groups and each group L on different processors are independent of each other the concurrent execution of evolutionary operation, after some evolution of every generation, each group will exchange between a number of individuals to introduce other subgroups of excellent genes, abundant diversity of each group, to prevent the occurrence of premature convergence Less communication overhead, at the same time, the coarse-grained model is very suitable for running on the communication bandwidth with low cluster system. Coordinate positioning is a commonly used to implement the wireless local area network (LAN) optimization techniques, it can make us efficient are multiple wireless access point of the environment, for covering the area of the user to provide more bandwidth and throughput. Coordinate positioning of the meaning is: one is to ensure that the transmission rate of the wireless local area network and equilibrium load; the second it is to avoid the adjacent channel interference and the same channel. Here introduces the coordinate location problem in wireless local area network (LAN) is mainly in order to avoid possible adjacent channel interference and the same channel. In the formula 7 8, we show the detail optimization process. In the figure 5, we show the basic pattern figure of optimizing.
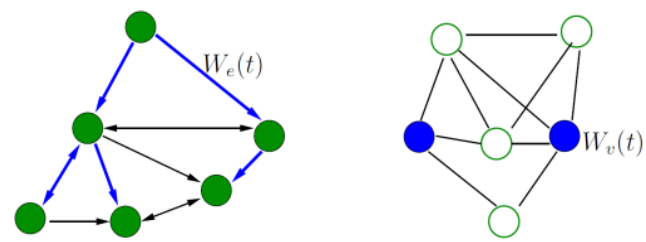

Figure 5.The Basic Pattern of the Optimization Process 


$$
\begin{aligned}
& R^{\pi}(T)=\square\left[\sum_{t=1}^{T}\left(<C(T), \mathrm{X}(\pi)>-<C(T) \cdot X^{*}>\right)\right] \\
& E(w, c)=\frac{1}{2} \sum_{p=1}^{P} \sum_{i=1}^{N}\left(w_{i} \ell\left(C_{i}\right)-y^{p}\right)^{2}+\frac{1}{2} \beta_{w}\|w\|^{2}
\end{aligned}
$$

Computer network service quality optimization is done by optimization algorithm, the optimization method can be divided into time and optimize the space, with the location of the different optimization algorithm, can be divided into centralized and distributed optimization, distributed optimization algorithm is done in the process of optimization to solve the optimization, the centralized optimization need controller, and using the computer for task scheduling and resource allocation optimization results, the two kinds of optimization method is optimized according to different network environment. Therefore, computer network optimization service measures, can adopt the centralized and distributed optimization was conducted in two ways, not only can reduce the network delay, resource scheduling task, also can ensure the accuracy of data exchange and quickness. The corresponding experimental simulation is shown in the following section.

\section{Experimental Analysis and Simulation}

Optimization of computer network service quality, the most basic problem mainly refers to the loss, network delay and data packet transmission errors, etc., if in the process of network transmission to transmit data to a buffer has been filled with a router, the router will only be able to according to the actual network status data transmission, and this will lose some data or the loss of all data, and the packet loss situation is unpredictable. And unable to advance forecast the transmission path of data packets, the packets could be met router congestion, and because the path different packets during transmission conditions and downs, due to the influence of different level. In the following figures, we illustrate the numerical analysis and corresponding simulation result for the proposed methodology.
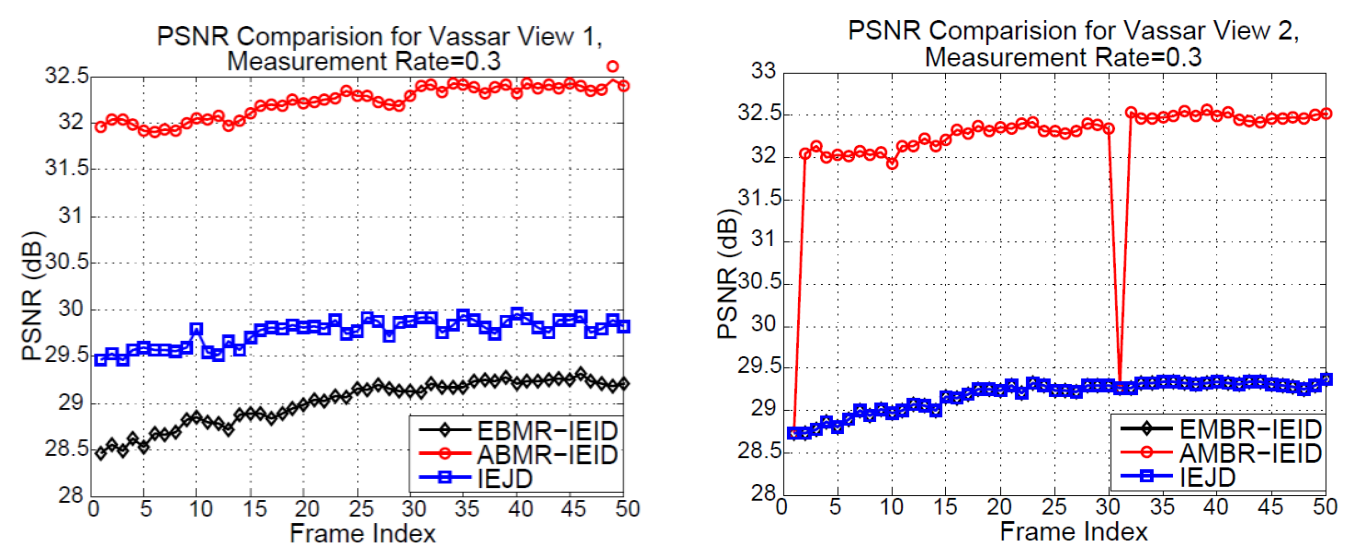

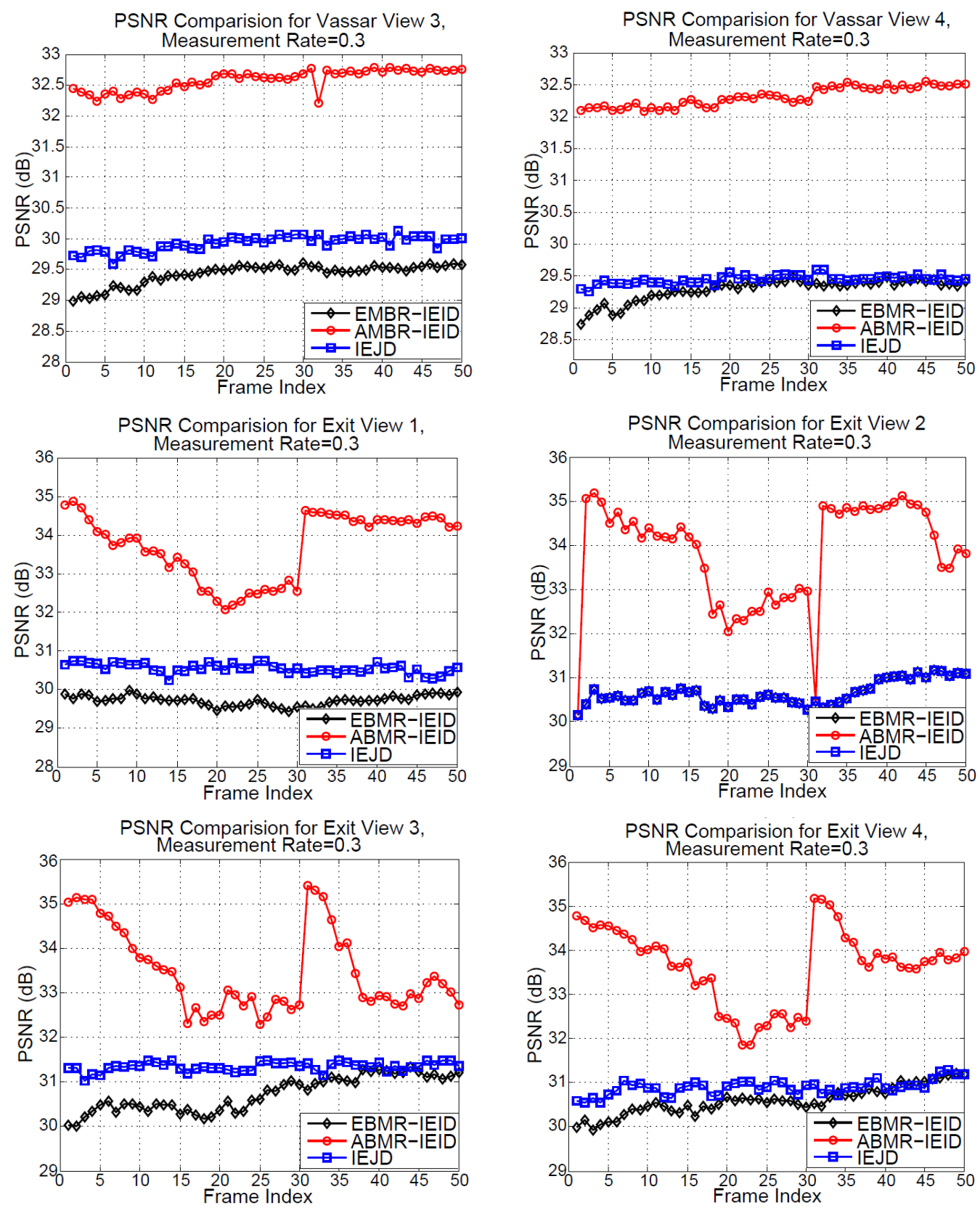

Figure 6.The Simulation Result for the Proposed Methodology

\section{Conclusion and Summary}

In this paper, we theoretically analysis the features of neural network structure and convex optimization related method. There are a lot of shortcomings but the cable network should be restricted the wiring in some situations, for example, wiring, reroute workload big, easy to damage, in the network, each node cannot move and so on. Our proposed methodology combines the advantages of neural network and convex optimization tool to obtain better result. The experiment result illustrate that our method outperforms compared with other state-of-the-art approaches. Inspire by the success of this approach, we plan to conduct more theoretical analysis on the novel pattern of optimizing. 


\section{References}

[1] T. Zhangxi, Z. Qian, X. Chen, K. Asanovic, and D. Patterson. "DIABLO: A Warehouse-Scale Computer Network Simulator using FPGAs." In Proceedings of the Twentieth International Conference on Architectural Support for Programming Languages and Operating Systems, pp. 207-221. ACM, (2015).

[2] B. Marek, and A. Paszkiewicz. "The Use of Statistical Signatures to Detect Anomalies in Computer Network." In Analysis and Simulation of Electrical and Computer Systems, pp. 251-260. Springer International Publishing, ( 2015).

[3] N. Bhargava Teja, N. Shibuya, A. Rodriguez, J. Tsay, J. Lopez, T. Nguyen, S. Zupancic, and D. YuChun Lie. "An Efficient and Robust Fall Detection System Using Wireless Gait Analysis Sensor with Artificial Neural Network (ANN) and Support Vector Machine (SVM) Algorithms." Open Journal of Applied Biosensor 3, no. 04 (2015): 29.

[4] S. Carson, and V. Geraymovych. "SYSTEMS AND METHODS FOR IMPLEMENTING COMPUTER SECURITY." U.S. Patent 20,150,026,767, issued Jan. 22,( 2015).

[5] W. Yujue, Q. Wu, B. Qin, X. Chen, X. Huang, and Y. Zhou. "Group-oriented Proofs of Storage." In Proceedings of the 10th ACM Symposium on Information, Computer and Communications Security, pp. 73-84. ACM, (2015).

[6] T. Diego, J. M. López-Lezama, M. J. Rider, and G. Vinasco. "Transmission network expansion planning considering repowering and reconfiguration." International Journal of Electrical Power \& Energy Systems 69 (2015), pp. 213-221.

[7] M. Ahmad, and N. L. Azad. "An Ensemble Neuro-Fuzzy Radial Basis Network with Self-Adaptive Swarm Based Supervisor and Negative Correlation for Modeling Automotive Engine Coldstart Hydrocarbon Emissions: A Soft Solution to a Crucial Automotive Problem." Applied Soft Computing (2015).

[8] S. Sudip, A. Adiga, B. Aditya Prakash, and A. Kumar S. Vullikanti. "Approximation Algorithms for Reducing the Spectral Radius to Control Epidemic Spread." arXiv preprint arXiv: pp. 1501.06614 (2015).

[9] E. Pfetsch, Marc., A. Fügenschuh, B. Geißler, N. Geißler, R. Gollmer, B. Hiller, J. Humpola et al. "Validation of nominations in gas network optimization: Models, methods, and solutions." Optimization Methods and Software 30, no. 1 (2015), pp. 15-53.

[10] Supittayapornpong, Sucha, and Michael J. Neely. "Achieving Utility-Delay-Reliability Tradeoff in Stochastic Network Optimization with Finite Buffers." arXiv preprint arXiv, pp. 1501.03457 (2015).

[11] T. Yutaka, T. Hashiguchi, K. Tajima, T. Katagiri, T. Naito, Q. Zhang, . Wang, Inwoong Kim, Paparao Palacharla, and Motoyoshi Sekiya. "Agile Network Re-optimization Supporting Seamless Service Migration." In Optical Fiber Communication Conference, pp. Tu3H-2. Optical Society of America, (2015).

[12] Y. Hongxue. "Design of a Python-based Wireless Network Optimization and Testing Sys-tem." Open Automation and Control Systems Journal 7 (2015), pp. 353-357.

[13] S. Angelo. "Classification of network optimization software packages." Encyclopedia of Information Science and Technology (3rd edition), IGI Global, Hershey, PA (2015), pp. 7054-7062.

[14] C., Antonio, M. Pioro, Y. Li, and D. Yuan. "On packet transmission scheduling for min-max delay and energy consumption in wireless mesh sensor networks." In INOC 2015, 7th International Network Optimization Conference. (2015).

[15] Z. Hang, and R. Jiang. "The Memetic algorithm for the optimization of urban transit network." Expert Systems with Applications 42, no. 7 (2015), pp. 3760-3773.

[16] W., Fei, Y. Xiong, and Z. Weng. "Neural Network Modeling of Submarine Shell." In Vibration Engineering and Technology of Machinery, pp. 1055-1064. Springer International Publishing, (2015).

[17] L. Byung-Yoon, S-M Hong, D-W Yoo, Hae-In Lee, Gun-Hee Moon, and Min-Jea Tahk. "Design of a Neural Network Controller for a Slung-Load System Lifted by 1 Quad-Rotor." Journal of Automation and Control Engineering, vol 3, no. 1, (2015).

[18] F. Segovia,., and C. Phillips. "PET imaging analysis using a parcelation approach and multiple kernel classification." In Pattern Recognition in Neuroimaging, 2014 International Workshop on, pp. 1-4. IEEE, (2014).

[19] C. Eduardo, V. Gómez-Verdejo, M. Martínez-Ramón, K. A. Kiehl, and V. D. Calhoun. "A multiple kernel learning approach to perform classification of groups from complex-valued fMRI data analysis: Application to schizophrenia." NeuroImage 87 (2014), pp. 1-17.

[20] H. Wang and J. Wang, "An effective image representation method using kernel classification," in 2014 IEEE 26th International Conference on Tools with Artificial Intelligence (ICTAI 2014), 2014, pp. 853858.

[21] C-V, Gustavo, D. Tuia, L. Bruzzone, and J. Atli Benediktsson. "Advances in hyperspectral image classification: Earth monitoring with statistical learning methods." Signal Processing Magazine, IEEE 31, no. 1 (2014), pp. 45-54.

[22] L. Wei, S. Prasad, and James E. Fowler. "Decision fusion in kernel-induced spaces for hyperspectral image classification." Geoscience and Remote Sensing, IEEE Transactions on 52, no. 6 (2014), pp. 3399-3411. 
[23] B. Soheil, N. M. Nasrabadi, A. Ray, and K. W. Jenkins. "Kernel Task-Driven Dictionary Learning for Hyperspectral Image Classification." arXiv preprint arXiv:1502.03126 (2015).

[24] J. Rongrong, Y. Gao, R. Hong, Q. Liu, D. Tao, and X. Li. "Spectral-spatial constraint hyperspectral image classification." Geoscience and Remote Sensing, IEEE Transactions on 52, no. 3 (2014), pp. 1811-1824.

[25] Z. De-Long, J. Qiao, J-B Li, L-Y Qiao, S-C Chu, and J. F. Roddick. "Optimizing matrix mapping with data dependent kernel for image classification." J. Inf. Hiding Multimed. Signal Process 5 (2014), pp. 72-79.

[26] Anaya, J. J., and D. Ruiz. "Network Optimization for Broadcast Contribution Services Under H. 264 Hi422 Profile." SMPTE Motion Imaging Journal 123, no. 3 (2014), pp. 49-55.

[27] H. MM Faruque, F. Boukouvala, Eric L. First, and Christodoulos A. Floudas. "Nationwide, Regional, and Statewide CO2 Capture, Utilization, and Sequestration Supply Chain Network Optimization." Industrial \& Engineering Chemistry Research 53, no.18(2014),pp.7489-7506. 
International Journal of Security and Its Applications

Vol.9, No.11 (2015) 